\title{
Clinical Presentation and Ischemic Zone on MRI in Cancer Patients with Acute Ischemic Stroke
}

\author{
Jin Myoung Seok ${ }^{a} \quad$ Suk Jae Kim ${ }^{a} \quad$ Pamela Song ${ }^{a, b} \quad$ Chin-Sang Chung ${ }^{a}$ \\ Gyeong-Moon Kim ${ }^{a}$ Kwang Ho Lee ${ }^{a}$ Oh Young Bang ${ }^{a}$ \\ ${ }^{a}$ Department of Neurology, Samsung Medical Center, Sungkyunkwan University School of Medicine, Seoul, and \\ ${ }^{b}$ Department of Neurology, Ilsan Paik Hospital, Inje University, Goyang, Korea
}

\section{Key Words}

Cancer $\cdot$ Stroke $\cdot$ Perfusion $\cdot$ Mismatch $\cdot$ Thrombolysis

\begin{abstract}
Aims: This study was conducted to evaluate the clinical and MRI profiles in acute cancer strokes, and to demonstrate our experience with thrombolytic therapy in cancer stroke patients. Methods: We prospectively studied active cancer patients with acute ischemic stroke who underwent MRI within $48 \mathrm{~h}$ of the onset of symptoms. Patients were grouped based on the presence of conventional stroke mechanisms (CSM). Clinical characteristics and MRI profiles were evaluated. $\boldsymbol{R e}$ sults: A total of 70 patients were finally included in this study. Patients without CSM were more frequently presented with encephalopathy than those with CSM ( 29.4 vs. $2.8 \%, p=$ 0.002). The diffusion-perfusion mismatch pattern was more prevalent in patients with CSM (21 patients, 58.3\%) than in patients without CSM (8 patients, $23.5 \%$ ). Patients who had a higher tertiles of $D$-dimer level were significantly less likely to have the diffusion-perfusion mismatch pattern $(p=0.015)$. Among patients who presented within $6 \mathrm{~h}$ of the onset of stroke, revascularization therapy was performed in 4 of 16 (25\%) patients with CSM, but none of the patients without
\end{abstract}

CSM. Conclusion: Based on the stroke mechanisms, the optimal strategy of thrombolytic therapy should be considered differently in cancer patients with acute ischemic stroke.

Copyright $\odot 2012$ S. Karger AG, Basel

\section{Introduction}

The proportion of patients who have cancer is expected to increase among stroke patients. The steadily increasing proportion of elderly people will result in an approximately $50 \%$ increase in new cancer cases worldwide over the next 20 years (World Health Organization, 2005). Moreover, the association between cancer and stroke has been reported [1-3]. However, treatment and preventive strategies for cancer-related stroke are unsettled. The use of thrombolytics within therapeutic time window is not contraindicated in stroke patients with cancer under the current guideline for acute stroke therapy [4]. Nevertheless, it is not known if acute thrombolytic treatments are appropriate for cancer patients with acute ischemic stroke.

We recently reported that cancer stroke patients without conventional stroke mechanisms (CSM) showed dif-

\section{KARGER}

Fax +4161306 1234 E-Mail karger@karger.ch www.karger.com
(C) 2012 S. Karger AG, Basel

0014-3022/12/0686-0368\$38.00/0

Accessible online at:

www.karger.com/ene
Oh Young Bang, $\mathrm{MD}, \mathrm{PhD}$

Department of Neurology and Brain and Nerve Center

Samsung Medical Center, Sungkyunkwan University School of Medicine

50 Irwon-dong, Gangnam-gu, Seoul 135-710 (Korea)

Tel. +82 23410 3599, E-Mail nmboy@unitel.co.kr 
ferent characteristics from those with CSMs [5], and that embolism by cancer-related coagulopathy is the main mechanism of stroke in patients without CSM [6]. Therefore, the clinical presentation of stroke and the eligibility or response of thrombolytic treatment may differ according to the stroke mechanisms. To our knowledge, this is the first report regarding clinical presentation and characteristics of ischemic zone specifically in stroke in cancer patients. Thus, we conducted this study to compare the clinical characteristics and MRI profiles (perfusiondiffusion and MRA-diffusion mismatch) of cancer patients with and without CSM.

\section{Methods}

\section{Patients}

We prospectively studied consecutive cancer patients with acute ischemic stroke registered at a university hospital between January 2006 and December 2009. Patients were identified as having (a) active cancer, excluding primary intracranial tumor, (b) suffered focal symptoms and undergone MRI within $48 \mathrm{~h}$ of symptom onset, (c) relevant lesions within the middle cerebral artery (MCA) distribution identified upon diffusion-weighted imaging (DWI) and (d) diagnostic workups, including vascular and cardiologic studies (see fig. 1 for patient selection). Active cancer was defined as a diagnosis of cancer within 6 months prior to enrollment, any treatment for cancer within the previous 6 months, or recurrent or metastatic cancer, as previously described [7]. The local institutional review boards approved the study, and all patients provided informed consent with respect to participation in this study.

MRI Methods and Imaging Analysis

All patients underwent DWI, PWI and magnetic resonance angiography (MRA) within $48 \mathrm{~h}$ using a 3.0T unit (Achieva, Philips Medical Systems, Best, The Netherlands). DWI was obtained with two levels of diffusion sensitization (b values of 0 and 1,000 $\mathrm{s} / \mathrm{mm}^{2}, 5-\mathrm{mm}$ slice thickness with $2-\mathrm{mm}$ interslice gap). PWI was conducted using gradient-echo and echo-planar sequences with the following parameters: TR, $1500 \mathrm{~ms}$; T3, $35 \mathrm{~ms}$; flip angle, $40^{\circ}$; matrix, $128 \times 128$; FOV $24 \mathrm{~cm}$; section thickness, $5 \mathrm{~mm}$, and intersection gap, $2 \mathrm{~mm}$. The PWI were processed to generate maps of the time-to-bolus peak (TTP), relative mean transit time, relative cerebral blood volume and relative cerebral blood flow, and the TTP maps were used to assess the perfusion status. DWI and PWI volume measurements were conducted by two of the authors (J.M.S. and S.J.K.) who were blinded to the clinical information. For each patient, the DWI and PWI lesion volumes were outlined manually, and the volumes were calculated using a computer-assisted volumetric analysis program (Medical Image Processing, Analysis and Visualization, version 2.1, CIT, NIH). The patients were categorized according to their baseline MRI profile. The Mismatch pattern was defined as a PWI lesion with a volume of $10 \mathrm{ml}$ or more and $120 \%$ or more of the DWI lesion. The small lesion pattern was defined as a DWI and PWI volume both less than $10 \mathrm{ml}$. The no mismatch pattern was defined as a PWI volume less than $120 \%$ of the DWI lesion volume [8].

Mismatch Profiles in Cancer-Related Ischemic Stroke
The MRA rating and MRA-DWI mismatch was defined according to the pre-specified MRA-DWI mismatch criteria [9]. For the intracranial ICA and M1 segment of the MCA: $1=$ normal, $2=$ reduced flow and $3=$ occlusion. For the M2 segment of the MCA: $1=$ normal and $2=$ abnormal. The MRA-DWI mismatch was defined as an MRA score of 3 and a DWI lesion volume $<25$ $\mathrm{ml}$ or an MRA score of 2 and a DWI lesion volume $<15 \mathrm{ml}$. The MRA-DWI mismatch was rated independently by two stroke neurologists (S.J.K. and O.Y.B.) with no clinical information. The third opinion (by G.M.K.) was obtained in cases of disagreement.

\section{Etiologic Workup and Characteristics of Stroke}

For all patients, age, gender and stroke risk factors were collected, and stroke workups including routine blood tests, electrocardiography, echocardiography and coagulation studies were conducted. All coagulation studies including D-dimer were examined within $24 \mathrm{~h}$ of the onset of stroke. Based on the results of vascular and cardiologic studies, the categories for the stroke mechanisms were assigned based on a modified TOAST classification, which includes large-artery atherosclerosis (LAA), cardioembolism (CE), small-vessel occlusion (SVO), other determined etiology, and unknown etiology [10]. We defined conventional stroke mechanism (CSM) as LAA, CE, SVO or other determined etiology. Patients were divided into two groups according to the presence of CSM: (1) patients with CSM and (2) patients without CSM.

The presenting clinical stroke syndromes were classified according to the modified Oxfordshire Community Stroke Project criteria as total anterior circulation syndrome (TACS), partial anterior circulation syndrome (PACS), lacunar syndrome (LACS), combined syndromes that localized to both the anterior and posterior circulations, and encephalopathy that showed mental status changes without documented focal neurological deficits [11, 12]. The severity of neurological deficits was assessed by the National Institute of Health Stroke Scale (NIHSS) score upon presentation.

\section{Thrombolytic Treatment Strategy}

The patients who presented within $6 \mathrm{~h}$ from the symptom onset were considered for the thrombolytic treatment in our institution. Patients who presented within $3 \mathrm{~h}$ from the symptom onset were treated with intravenous thrombolytic therapy unless contraindicated [4]. Among patients who presented between 3 and $6 \mathrm{~h}$ from the symptom onset, intra-arterial thrombolytic therapy were done when large artery occlusion or stenosis are documented in CT, MRI or conventional angiography [13].

\section{Statistical Analysis}

Continuous data are shown as the mean $\pm \mathrm{SD}$, whereas categorical variables are presented as absolute and relative frequencies. We analyzed the differences between patients with and without CSM and among groups stratified based on the tertiles of the D-dimer level using the Student's t test, the Mann-Whitney U test or the Kruskal-Wallis test for continuous variables, and the $\chi^{2}$ test, the Fisher exact test or the linear-by-linear association test for categorical variables. A value of $p<0.05$ was considered significant. All statistical analyses were conducted using commercially available software (SPSS for Windows, version 15.0; SPSS Inc., Chicago, Ill., USA). 
Fig. 1. Selection of patients.

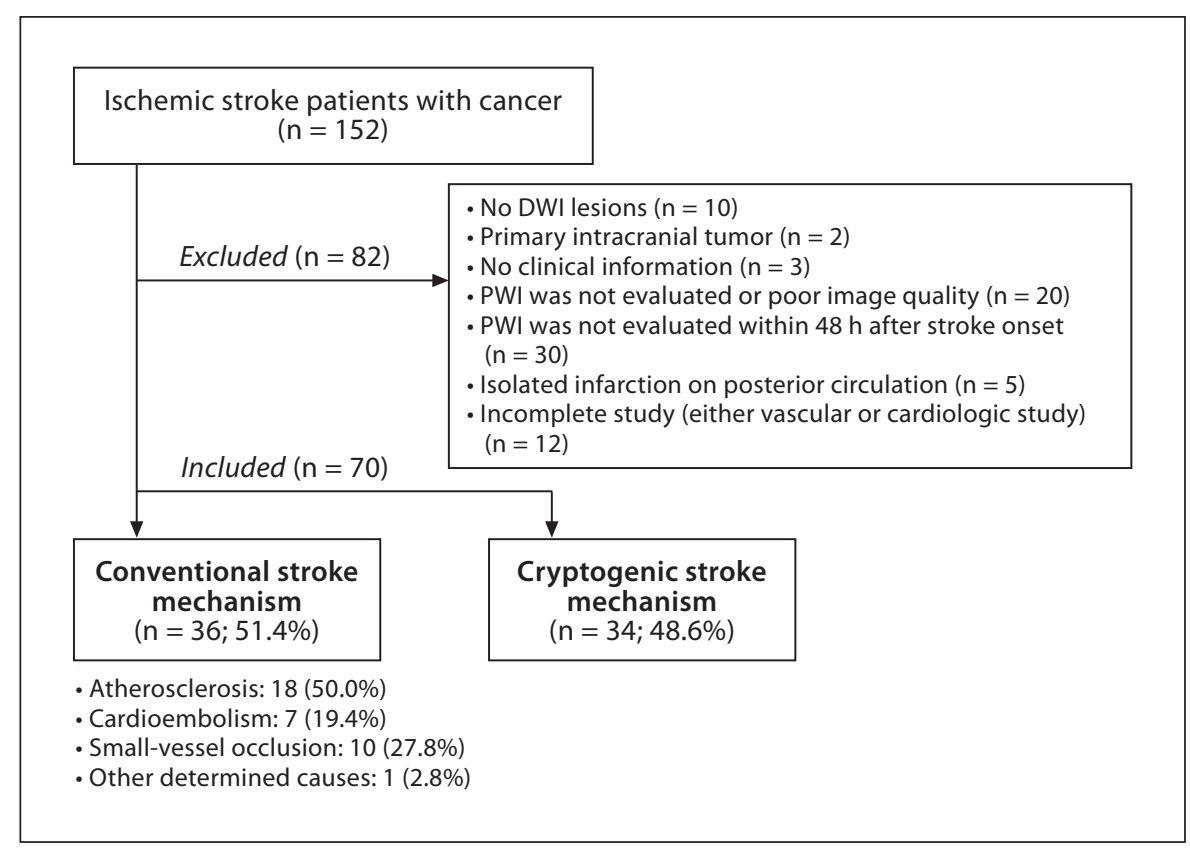

\section{Results}

Of 152 cancer patients with acute ischemic stroke, 70 (42 men and 28 women; age, $65.6 \pm 11.4$ years) were included in this study (fig. 1). Of the enrolled patients, 36 (51.4\%) had CSM and 34 (48.6\%) did not.

\section{Clinical Characteristics}

The clinical characteristics are shown in table $1 . \mathrm{Pa}-$ tients with CSM were significantly older than those without CSM, and the risk factors for stroke were more prevalent in patients with CSM than in those without CSM.

The NIHSS upon admission was $7.5 \pm 6.7$ and the time interval between symptom onset and time of presentation at the emergency room was $10.1 \pm 11.0 \mathrm{~h}$. Neither of these values differed significantly between groups. However, the presenting clinical stroke syndromes differed between groups, with the combined syndrome or encephalopathy being more frequently observed in patients without CSM than those with CSM (10 of 34 patients, $29.4 \%$ vs. 1 of 36 patients, $2.8 \% ; \mathrm{p}=0.002$ ), and there was a tendency for TACS to occur more frequently in patients with the CSM than in those without CSM (7 of 36 patients, $19.4 \%$ vs. 2 of 34 patients, $5.9 \%$; $p=0.152$ ). A sample case of cancer stroke without CSM is shown in figure 2 .

\section{MRI Profiles}

The time interval between symptom onset and MRI study was $15.4 \pm 13.0 \mathrm{~h}$, and this value did not differ between groups. However, the MRI profile was significantly different. The mismatch pattern was more prevalent in patients with CSM (21 patients, 58.3\%) than in those without CSM (8 patients, 23.5\%) ( $\mathrm{p}<0.001)$. Conversely, the no mismatch pattern was more prevalent in patients without CSM (23 patients, 67.6\%) than in patients with CSM (6 patients, 16.7\%). Similarly, MRA-DWI mismatch was more frequently observed in patients with CSM than in those without CSM (77.8 vs. 9.7\%; $\mathrm{p}<0.001)$.

\section{Association between D-Dimer and Clinical and MRI Characteristics}

Coagulation studies revealed that $\mathrm{D}$-dimer levels were higher in patients without CSM than in those with CSM $(16.06 \pm 22.40 \mu \mathrm{g} / \mathrm{ml}$ vs. $4.67 \pm 8.77 \mu \mathrm{g} / \mathrm{ml} ; \mathrm{p}=0.001)$ (table 1). Therefore, we evaluated the association between D-dimer levels and clinical and MRI characteristics. All patients were stratified into three groups according to inter-tertile cutoff points of the distribution of $\mathrm{D}$-dimer levels (table 2). The time intervals between symptom onset and MRI scanning and between symptom onset and time of presentation did not differ significantly among groups stratified based on the $\mathrm{D}$-dimer levels.

Patients who had a higher D-dimer level were significantly more likely to have the no mismatch pattern $(\mathrm{p}=$ 
Table 1. Clinical, laboratory characteristics and MR imaging profiles in ischemic stroke patients with cancer

\begin{tabular}{|c|c|c|c|c|}
\hline & Total $(\mathrm{n}=70)$ & $\begin{array}{l}\text { Patients with CSM } \\
(\mathrm{n}=36 ; 51.4)\end{array}$ & $\begin{array}{l}\text { Patients without CSM } \\
(\mathrm{n}=34 ; 48.6)\end{array}$ & $\mathrm{p}$ \\
\hline Age, years (SD) & $65.6(11.4)$ & $68.1(12.3)$ & $62.8(9.8)$ & 0.026 \\
\hline Male gender & $42(60.0)$ & $25(69.4)$ & $17(50.0)$ & 0.097 \\
\hline \multicolumn{5}{|l|}{ Risk factors } \\
\hline Hypertension & $30(42.9)$ & $20(55.6)$ & $10(29.4)$ & 0.027 \\
\hline Diabetes mellitus & $15(21.4)$ & $5(13.9)$ & $10(29.4)$ & 0.114 \\
\hline Hyperlipidemia & $12(17.1)$ & $7(19.4)$ & $5(14.7)$ & 0.599 \\
\hline Atrial fibrillation & $7(10.0)$ & $7(19.4)$ & 0 & 0.011 \\
\hline Ischemic heart disease & $5(7.1)$ & $4(11.1)$ & $1(3.0)$ & 0.359 \\
\hline Smoking & $18(25.7)$ & $9(25.0)$ & $9(26.5)$ & 0.888 \\
\hline \multicolumn{5}{|l|}{ Clinical presentation } \\
\hline $\begin{array}{l}\text { Mean time interval between symptom onset } \\
\text { and time of presentation, } \mathrm{h}(\mathrm{SD})\end{array}$ & $10.1(11.0)$ & $8.9(8.8)$ & $11.4(12.9)$ & 0.403 \\
\hline Initial NIHSS on admission (SD) & $7.5(6.7)$ & $8.0(7.3)$ & $6.7(6.0)$ & 0.374 \\
\hline \multicolumn{5}{|l|}{ The presenting clinical stroke syndromes } \\
\hline TACS & $9(12.9)$ & $7(19.4)$ & $2(5.9)$ & \\
\hline PACS & $31(44.3)$ & $16(44.4)$ & $15(44.1)$ & \\
\hline LACS & $19(27.1)$ & $12(33.3)$ & $7(20.6)$ & \\
\hline Combined/encephalopathy & $11(15.7)$ & $1(2.8)$ & $10(29.4)$ & 0.002 \\
\hline \multicolumn{5}{|l|}{ MRI profiles } \\
\hline \multicolumn{5}{|l|}{ Mean time interval between symptom onset } \\
\hline PWI-DWI mismatch profiles & & & & $<0.001$ \\
\hline Small lesion & $12(17.1)$ & $9(25.0)$ & $3(8.8)$ & \\
\hline No mismatch & $29(41.4)$ & $6(16.7)$ & $23(67.6)$ & \\
\hline Mismatch & $29(41.4)$ & $21(58.3)$ & $8(23.5)$ & \\
\hline MRA-DWI mismatch ${ }^{\mathrm{a}}$ & $24 / 58(41.4)$ & $21 / 27(77.8)$ & $3 / 31(9.7)$ & $<0.001$ \\
\hline \multicolumn{5}{|l|}{ Coagulation study } \\
\hline Mean D-dimer, $\mu \mathrm{g} / \mathrm{ml}(\mathrm{SD})$ & $10.45(17.91)$ & $4.67(8.77)$ & $16.06(22.40)$ & 0.001 \\
\hline Mean fibrinogen, mg/dl (SD) & $351.8(184.0)$ & $369.6(126.3)$ & $331.7(233.7)$ & 0.053 \\
\hline
\end{tabular}

0.015). Similarly, there was a tendency for patients with a higher D-dimer level to be less likely to have MRA-DWI mismatch, although this difference was not statistically significant $(p=0.104)$. Conversely, patients with a higher $\mathrm{D}$-dimer level tended to have encephalopathy rather than classical stroke syndromes as an acute presenting symptom, although this difference was not statistically significant $(\mathrm{p}=0.111)$.

\section{Thrombolytic Therapy Use}

Table 3 shows the acute therapy and reasons not to use revascularization therapy. Among 29 patients who presented within $6 \mathrm{~h}$ of the onset of stroke, only 4 patients were treated with revascularization therapy. All of the patients treated with revascularization therapy had CSM, 2 of these patients received intravenous thrombolysis, and the other two were treated with intra-arterial thrombolytic therapy. They showed good short-term clinical outcomes, with an average mean improvement of $3.75 \pm 1.7$ points in the NIHSS score 1 week after thrombolytic therapy, without any hemorrhage. None of patients without CSM were treated with revascularization therapy.

Revascularization therapy was not used for various reasons. In most patients with CSM (9 of 12 patients, $75 \%)$, recanalization therapy was not used due to mild stroke and rapid improvement, whereas in most patients without CSM (8 of 13 patients, 61.5\%) recanalization therapy was contraindicated due to medical conditions such as thrombocytopenia or prolonged INR related to cancer per se or cancer therapy. 


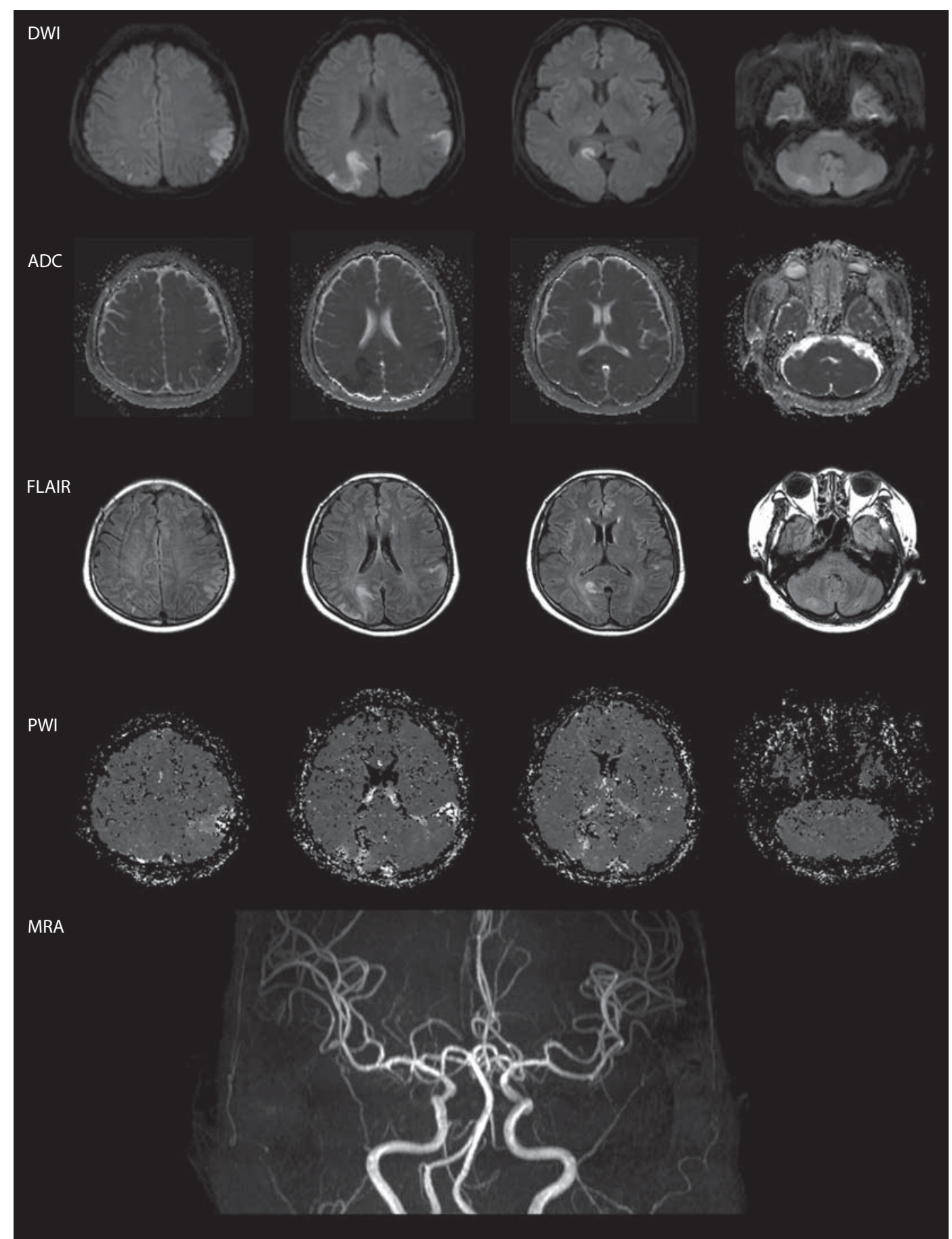

Fig. 2. A case of atypical presentation of stroke in cancer patients without conventional stroke mechanism. A 61-year-old female patient was diagnosed with multiple metastasis involving her liver, pancreas and lymph nodes, of unknown origin. One week before admission she experienced transient ataxia and memory impairment. Two days before admission her family found that she had difficulty in calculation. She was admitted for liver biopsy. After admission, she was found to have acute neurological symptoms of dysarthria and sleeping tendency. Neurological examination revealed drowsy mental status and poor obey command response, but normal motor and sensory response to pain stimulus. DWI showed high signal changes in the middle cerebral and posterior cerebral artery territories and cerebellum with various sizes. The PWI was normal except in the area in which there were signal changes in DWI, and MRA revealed no stenosis. The echocardiography results were normal. The initial D-dimer level of $66.8 \mu \mathrm{g} / \mathrm{ml}$ decreased to 12.9 $\mu \mathrm{g} / \mathrm{ml}$ with anticoagulation. The liver biopsy was pathologically confirmed as metastatic adenocarcinoma, which originated from the pancreas. 
Table 2. Association between D-dimer (tertiles) and clinical and MRI characteristics in cancer patients with acute ischemic stroke

\begin{tabular}{|c|c|c|c|c|}
\hline & \multicolumn{3}{|c|}{ D-dimer level (tertiles) } & \multirow[t]{2}{*}{$\mathrm{p}$} \\
\hline & $\begin{array}{l}\mathrm{T} 1(<1.14 \mu \mathrm{g} / \mathrm{ml}) \\
(\mathrm{n}=22 ; 33.8)\end{array}$ & $\begin{array}{l}\text { T2 }(1.14-5.40 \mu \mathrm{g} / \mathrm{ml}) \\
(\mathrm{n}=21 ; 32.3)\end{array}$ & $\begin{array}{l}\text { T3 }(>5.40 \mu \mathrm{g} / \mathrm{ml}) \\
(\mathrm{n}=22 ; 33.8)\end{array}$ & \\
\hline \multicolumn{5}{|l|}{ MRI profiles } \\
\hline \multicolumn{5}{|l|}{ PWI-DWI mismatch profiles } \\
\hline Small lesion & $9(40.9)$ & $2(9.5)$ & 0 & \\
\hline No mismatch & $6(27.3)$ & $6(28.6)$ & $14(63.6)$ & 0.015 \\
\hline Mismatch & $7(31.8)$ & $13(61.9)$ & $8(36.4)$ & \\
\hline MRA-DWI mismatch ${ }^{\mathrm{a}}$ & $8 / 13(61.5)$ & $7 / 19(36.8)$ & $7 / 22(31.8)$ & 0.104 \\
\hline $\begin{array}{l}\text { Mean time interval between symptom } \\
\text { onset and MRI study, h (SD) }\end{array}$ & $18.0(13.2)$ & $11.8(12.6)$ & $16.3(12.5)$ & 0.225 \\
\hline \multicolumn{5}{|l|}{ Clinical presentation } \\
\hline $\begin{array}{l}\text { Mean time interval between symptom } \\
\text { onset and time of presentation, } h(S D)\end{array}$ & $10.4(9.8)$ & $9.4(12.2)$ & $10.7(10.7)$ & 0.511 \\
\hline \multicolumn{5}{|l|}{ The presenting clinical stroke syndromes } \\
\hline TACS & $2(9.1)$ & $2(9.5)$ & $5(22.7)$ & \\
\hline PACS & $8(36.4)$ & $12(57.1)$ & $8(36.4)$ & \\
\hline LACS & $10(45.5)$ & $4(19.0)$ & $3(13.6)$ & \\
\hline Combined/encephalopathy & $2(9.1)$ & $3(14.3)$ & $6(27.3)$ & 0.111 \\
\hline
\end{tabular}

${ }^{\text {a }}$ Patients with small lesion profile were excluded in the analysis. Unless otherwise stated, values in parentheses denote percentages.

Table 3. Acute therapy and reasons for not using revascularization therapy in patients who present within $6 \mathrm{~h}$ after symptom onset

\begin{tabular}{|c|c|c|c|}
\hline & $\begin{array}{l}\text { Patients with } \\
\text { CSM }(\mathrm{n}=16)\end{array}$ & $\begin{array}{l}\text { Patients without } \\
\text { CSM }(n=13)\end{array}$ & $\mathrm{p}$ \\
\hline Thrombolytic treatment & & & 0.107 \\
\hline No thrombolytic therapy use & $12(75.0)$ & $13(100)$ & 0.066 \\
\hline 1 Not indicated for thrombolytic treatment & $9(75.0)$ & $5(38.5)$ & \\
\hline Mild stroke severity (NIHSS $<4)$ & 8 & 5 & \\
\hline Rapid improvement & 1 & 0 & \\
\hline 2 Contraindications of thrombolytic treatment & $3(25.0)$ & $8(61.5)$ & \\
\hline Thrombocytopenia (platelet count $<100,000 / \mathrm{mm}^{3}$ ) & 0 & 4 & \\
\hline Prolonged INR $($ INR $>1.7)$ & 0 & $1^{\mathrm{a}}$ & \\
\hline Large lobar infarction (hypodensity $>1 / 3$ cerebral hemisphere on CT scan) & 1 & 0 & \\
\hline Refusal by the family member & 1 & 1 & \\
\hline No demonstrable large vessel occlusion ${ }^{\mathrm{b}}$ & 1 & 2 & \\
\hline Thrombolytic therapy use & $4(25.0)$ & 0 & \\
\hline
\end{tabular}

a The patient was on warfarin treatment due to previous pulmonary thromboembolism.

${ }^{\mathrm{b}}$ Ineligible for intra-arterial thrombolytic therapy due to no documented large vessel occlusion, although patients were presented within 3-6 h after the onset of stroke. Values in parentheses denote percentages. 


\section{Discussion}

The results of the present study showed that in the acute setting the clinical and MR imaging characteristics of cancer patients with ischemic stroke varied depending on the mechanism of stroke. Specifically, cancer patients with acute stroke caused by hypercoagulability showed distinct clinical presenting stroke syndromes and ischemic zones from those with CSM.

Within minutes of an ischemic insult, a core region of tissue exhibits profound loss of blood flow and becomes irreversibly damaged, even if blood flow is rapidly restored. The surrounding penumbra zone may still be rescued for several hours or more after symptom onset. Thus, identification of ischemic penumbra in acute ischemic stroke is important because the ischemic penumbra represents a suitable target for revascularization therapy. The most common technique for evaluating the ischemic zone is combined PWI and DWI $[8,14]$ or MRA and DWI [9]. However, no efforts have been made to evaluate the characteristics of ischemic zone in cancer patients with acute stroke. To the best of our knowledge, this report is the first clinical study conducted to evaluate MRI (PWI-DWI and MRA-DWI) mismatch profiles in cancer patients with acute ischemic stroke. The data presented herein showed that when patients were divided based on the presence of CSM, the mismatch profiles differed greatly. The mismatch pattern was the main feature in patients with CSM, being observed in $58.3 \%$ of patients. This result is similar to those that have previously been reported in ischemic stroke patients without cancer of $48 \%$ in the DEFUSE trial and $51 \%$ in the EPITHET trial $[8,15]$. However, the mismatch pattern was a minor feature in patients without CSM, and most patients without CSM had no mismatch pattern. The MRA-DWI mismatch showed similar findings, with patients without CSM having the MRA-DWI mismatch less often than those with CSM, or the result of previous study by Lansberg et al. [9]. These results suggest that patients with the cancer-related stroke mechanism often show normal perfusion status and angiographic findings.

In the present study, we also evaluated the clinical characteristics of cancer stroke patients. Most of the clinical features were not obviously different, and the overall stroke severity evaluated by NIHSS was moderate and did not differ between groups. Interestingly, in the present study, encephalopathy syndrome on presentation was common in patients without CSM. Approximately $30 \%$ of patients without CSM presented with progressive neu- rological deficits over hours to days rather than sudden focal events and showed no focal neurological signs other than dysarthria and altered mental status. These patients can often be misdiagnosed as having medical complications or metastasis to the brain. Thus, one should keep in mind that patients with active cancer atypical presentation of acute stroke should be considered when patients showed diffuse cerebral dysfunction or minor progressive symptoms.

Our data showed that coagulopathy was associated with the perfusion status and acute presenting stroke syndromes. In our study, patients with a higher D-dimer level had a normal perfusion status more often than those with lower $\mathrm{D}$-dimer levels. The $\mathrm{D}$-dimer is a direct measure of activated coagulation and fibrinolysis that is widely used as a measure of hypercoagulability in cancer patients $[16,17]$. It is well known that higher $\mathrm{D}$-dimer levels are related to the stroke severity and the acute phase of ischemic stroke $[18,19]$. But in our study, all D-dimer studies were evaluated within $24 \mathrm{~h}$ of the onset of stroke and there was no significant correlation between D-dimer and initial NIHSS (Spearman correlation coefficient $=0.169, \mathrm{p}=0.185$ ). Thus, we believe that the levels of $\mathrm{D}$-dimer were not largely influenced by those factors. Hypercoagulability was proposed as one of the main mechanisms of ischemic stroke in cancer patients. A previous study of cancer stroke by Cestari et al. showed the possibility of hypercoagulability-related cancer stroke using the D-dimer level [20], and we recently reported that a high $\mathrm{D}$-dimer level could predict the cancer stroke without CSM (possibly cancer-related stroke) and the presence of embolic signals upon transcranial Doppler study, which represents embolism $[5,6]$. Therefore, it is conceivable that a higher D-dimer level in cancer stroke may represent cancer-related stroke, which has normal perfusion status but multiple embolisms due to intravascular coagulopathy and is not a suitable target of thrombolytic treatment. The proper prevention of recurrent embolism may be more important in such patients. A dramatic recovery with appropriate preventive strategies could be expected because patients with cancer-related stroke mechanism often show multiple scattered lesions [5] and a higher rate of recurrent embolism [6].

During the 4-year study period, only 4 patients with CSM and no patients without CSM were treated with thrombolytic therapy. Our results showed that although patients who were treated with thrombolytic therapy may show favorable responses even though they had active cancer, recanalization therapy was often contraindi- 
cated in patients without CSM. We recently showed the possibility of anticoagulant use for treatment of cancerrelated strokes [6]. The levels of D-dimer dramatically decreased with the use of anticoagulants in most patients, suggesting the importance of anticoagulants over recanalization therapy in acute stroke patients without CSM. To confirm this, further controlled clinical studies of cancer stroke patients considering stroke treatment are needed.

The strengths of this study include consecutive recruitment of patients with comprehensive evaluations of stroke mechanisms and mismatch profiles using two different definitions. However, this study has several limitations. First, a TTP map was used to identify PWI-DWI mismatch. A multiparameter approach may help define the ischemic zone by combining data from various PWI parameters. No parameters among various perfusion parameters are superior for predicting tissue fate after recanalization, and the size of a perfusion lesion differs markedly among various perfusion parameters [21]. However, the distribution of the mismatch profile of our patients with CSM was similar to those of previous studies $[8,9,15]$. Second, although all the included patients had full stroke work-ups including trans-thoracic echocardiography, not all patients underwent trans-esophageal echocardiography (14 of 70 patients in total; 5 of 36 (13.9\%) patients with CSM and 9 of 34 (26.5\%) patients without CSM). Trans-esophageal echocardiography was performed in selected cases because of coagulopathy, bleeding tendency, mental changes or acute illness due to systemic cancer or stroke itself. Third, the data evaluated here were from a unique population, Korean patients with cancer. Accordingly, further investigations of different study populations are warranted.

In conclusion, considering the characteristics of acute presenting symptoms and the ischemic zone assessed by MRI in cancer-related stroke, acute stroke management in clinical practice should be tailored to the stroke mechanisms in each patient. Patients with cancer-related stroke are often ineligible for recanalization therapy due to coexistence of contraindications or because they are unlikely to show a favorable response to recanalization therapy due to the absence of penumbra at the time of presentation of ischemic symptoms. Strategies for acute stroke treatment might focus on the correction of coagulopathy rather than the resolution of mismatch. Conversely, stroke patients with CSMs could be treated like noncancer patients.

\section{Acknowledgements}

This study was supported by a grant from the Korean Healthcare Technology R\&D Project, Ministry of Health \& Welfare (A110208).

\section{Disclosure Statement}

None.

\section{References}

1 Graus F, Rogers LR, Posner JB: Cerebrovascular complications in patients with cancer. Medicine (Baltimore) 1985;64:16-35.

$\checkmark 2$ Bick RL: Cancer-associated thrombosis. N Engl J Med 2003;349:109-111.

>3 Rogers LR: Cerebrovascular complications in patients with cancer. Semin Neurol 2004; 24:453-460.

-4 Adams HP Jr, del Zoppo G, Alberts MJ, et al: Guidelines for the early management of adults with ischemic stroke: a guideline from the American Heart Association/American Stroke Association Stroke Council, Clinical Cardiology Council, Cardiovascular Radiology and Intervention Council, and the Atherosclerotic Peripheral Vascular Disease and Quality of Care Outcomes in Research Interdisciplinary Working Groups: the American Academy of Neurology affirms the value of this guideline as an educational tool for neurologists. Stroke 2007;38:1655-1711.
5 Kim SG, Hong JM, Kim HY, et al: Ischemic stroke in cancer patients with and without conventional mechanisms: a multicenter study in Korea. Stroke 2010;41:798-801.

6 Seok JM, Kim SG, Kim JW, et al: Coagulopathy and embolic signal in cancer patients with ischemic stroke. Ann Neurol 2010;68: 213-219.

7 Lee AY, Levine MN, Baker RI, et al: Low-molecular-weight heparin versus a coumarin for the prevention of recurrent venous thromboembolism in patients with cancer. N Engl J Med 2003;349:146-153.

8 Albers GW, Thijs VN, Wechsler L, et al: Magnetic resonance imaging profiles predict clinical response to early reperfusion: the diffusion and perfusion imaging evaluation for understanding stroke evolution (DEFUSE) study. Ann Neurol 2006;60:508517.
-9 Lansberg MG, Thijs VN, Bammer R, et al: The MRA-DWI mismatch identifies patients with stroke who are likely to benefit from reperfusion. Stroke 2008;39:2491-2496.

10 Adams HP Jr, Bendixen BH, Kappelle LJ, et al: Classification of subtype of acute ischemic stroke. Definitions for use in a multicenter clinical trial. TOAST. Trial of Org 10172 in Acute Stroke Treatment. Stroke 1993;24:35-41.

11 Bamford J, Sandercock P, Dennis M, Burn J, Warlow C: Classification and natural history of clinically identifiable subtypes of cerebral infarction. Lancet 1991;337:15211526.

12 Singhal AB, Topcuoglu MA, Buonanno FS: Acute ischemic stroke patterns in infective and nonbacterial thrombotic endocarditis: a diffusion-weighted magnetic resonance imaging study. Stroke 2002;33: 1267-1273. 
13 Choi JH, Bateman BT, Mangla S, et al: Endovascular recanalization therapy in acute ischemic stroke. Stroke 2006;37:419-424.

$\checkmark 14$ Kidwell CS, Alger JR, Saver JL: Beyond mismatch: evolving paradigms in imaging the ischemic penumbra with multimodal magnetic resonance imaging. Stroke 2003;34: 2729-2735.

15 Davis SM, Donnan GA, Parsons MW, et al: Effects of alteplase beyond $3 \mathrm{~h}$ after stroke in the Echoplanar Imaging Thrombolytic Evaluation Trial (EPITHET): a placebo-controlled randomised trial. Lancet Neurol 2008;7:299-309.
6 Wells PS, Anderson DR, Rodger M, et al: Evaluation of D-dimer in the diagnosis of suspected deep-vein thrombosis. N Engl J Med 2003;349:1227-1235.

17 Wada H, Kobayashi T, Abe Y, et al: Elevated levels of soluble fibrin or D-dimer indicate high risk of thrombosis. J Thromb Haemost 2006;4:1253-1258.

18 Feinberg WM, Erickson LP, Bruck D, Kittelson J: Hemostatic markers in acute ischemic stroke. Association with stroke type, severity, and outcome. Stroke 1996;27:1296-1300.

19 Tombul T, Atbas C, Anlar O: Hemostatic markers and platelet aggregation factors as predictive markers for type of stroke and neurological disability following cerebral infarction. J Clin Neurosci 2005;12:429-434.
20 Cestari DM, Weine DM, Panageas KS, Segal AZ, DeAngelis LM: Stroke in patients with cancer: incidence and etiology. Neurology 2004;62:2025-2030.

-21 Kane I, Carpenter T, Chappell F, et al: Comparison of 10 different magnetic resonance perfusion imaging processing methods in acute ischemic stroke: effect on lesion size, proportion of patients with diffusion/perfusion mismatch, clinical scores, and radiologic outcomes. Stroke 2007;38:3158-3164. 